\title{
Interface dynamics from experimental data
}

\author{
Achille Giacometti ${ }^{(1)}$, and Maurice Rossi ${ }^{(2)}$ \\ (1) INFM Unitá di Venezia, Dipartimento di Scienze Ambientali, Universitá di Venezia, \\ Calle Larga Santa Marta DD 2137, I-30123 Venezia-Italy \\ (2) Laboratoire de Modélisation en Mécanique, Université de Paris VI, 4 Place Jussieu, F-75252 Paris Cedex 05, France
}

(November 21, 2018)

\begin{abstract}
A novel algorithm is envisaged to extract the coupling parameters of the Kardar-Parisi-Zhang (KPZ) equation from experimental data. The method hinges on the Fokker-Planck equation combined with a classical least-square error procedure. It takes properly into account the fluctuations of surface height through a deterministic equation for space correlations. We apply it to the $1+1 \mathrm{KPZ}$ equation and carefully compare its results with those obtained by previous investigations. Unlike previous approaches, our method does not require large sizes and is stable under a modification of sampling time of observations. Shortcomings associated to standard discretizations of the continuous KPZ equation are also pointed out and possible future perspectives are finally analyzed.
\end{abstract}

64.60.Ht,05.40.+j,05.70.Ln

\section{INTRODUCTION}

Inverse techniques have a wide range of applicability ranging from geophysics to nonlinear time analysis and statistics [1]. The common philosophy behind these methods is the extraction of equations of motion starting from successive experimental time series of some dynamical variable in addition to basic assumptions such as determinism. If a reasonably general form of the equations is guessed either by symmetry arguments or by general considerations, the "true" parameters are then determined by minimizing a cost function quantifying the distance between experimental observations and corresponding reconstructed quantities, the latter being implicitly dependent upon the parameters. Among such approaches, the Least Square method is the most popular one.

A typical system which can be treated using reconstruction techniques is the case where an observational noise is superimposed to a standard deterministic evolution. In this case the system is expected to evolve under the action of deterministic system and stochasticity come only from our measurement apparatus. The particular case where the dynamics underlying the system is chaotic has also received considerable attention due to its widespread occurence in natural systems [2], and the importance of treating the presence of the noise with due care has already been emphasized [3].

The alternative possibility of dynamical noise occurs whenever the noise is a built-in component of the equations of motion. This is a far more difficult problem since one has to deal with stochastic rather than deterministic equations. An important such case, which is rather ubiquitous in nature, is the Langevin dynamics where variables evolve subject both to dissipative generalized forces and to a fluctuating part [1]. In this latter instance, the presence of dynamical noise can drastically modify the dynamics and hence hampers the efficiency of the usual reconstruction techniques based on deterministic ideas [5].

In our work, we focus on a particular class of Langevin dynamics which has its origin in a seminal paper on interface dynamics [6] but has ever since displayed relations to a variety of physical systems such as for instance bacterial colonial growth, immiscible fluids, directed polymer and superconductors [7].

The Kardar-Parisi-Zhang (KPZ) equation [6] was introduced as a coarse-grained mesoscopic description for the growth of a rough surface under the deposition of particles driven by gravity. The crucial ingredient introduced in the KPZ and not present in the corresponding linear counterpart, namely the Edward-Wilkinson (EW) equation [8], is a non-linear term which takes into account the fact that the growth is normal to the surface. The KPZ equation can be mapped into various other models. A Cole-Hopf change of variables maps it into a directed polymer diffusion equation subject to a random potential [9], while the identification of the local gradient with a velocity leads to the Burgers's equation for a vorticityfree velocity field [10. Furthermore it is believed that the KPZ equation has the same large-scale behavior of the Kuramoto-Sivashinsky equation in $1+1$ dimensions [11], while in higher dimensionality the situation is much less clear [12]. Nonetheless, in spite of the gigantic effort devoted to the KPZ equation in the past decade, a complete understanding of its properties is still lacking.

The aim of the present paper is to introduce a new inverse approach to the KPZ equation. A previous attempt due to Lam and Sander 13 was based on the standard Least Square (LS) reconstruction method. These authors used this approach directly on numerically simulated experimental surfaces without a preventive test of the performance of the method itself. Lam and Shin [14] subsequently showed that the standard discretizations used in 13 was not adequate. We shall argue below that, even with the improvements given in [14], the classical identification procedure devised in Ref. [13] is not properly suited for Langevin dynamics since it is based on deterministic equation ideas. By an explicit computation 
using the LS technique applied to a $1+1 \mathrm{KPZ}$ equation, we shall review their method and point out what we consider its main deficiencies.

We then go on to introduce a different approach based on the Fokker-Plank equation (FPE) associated to each Langevin equation [15], [4]. The advantage of this viewpoint is that one can construct deterministic relations among correlation functions which however still carry informations regarding the fluctuating nature of the original quantities. Those equations can then be easily analyzed within a least squares framework as in the LS method.

The paper is organized as follows. In Sec. II, the $\mathrm{KPZ}$ equation is rapidly recalled along with its numerical real space approximations in $1+1$ dimensions while the LS approach is reviewed in section III. Sec. IV contains the basic equations of our modified method which is then applied in Sec. V. Numerical results are then given in Sec. VI and some concluding remarks are provided in Sec. VII. More technical points are finally confined in the Appendices. Appendix A shows why the least square method fails for sufficiently large noise amplitudes and Appendix B presents some results concerning renormalized interfaces and their corresponding renormalized equations.

\section{INTERFACE DYNAMICS}

We consider a one-dimensional line of total length $L$ and a surface of height $h(x, t)$ at position $x$ and time $t$. The continuum $1+1 \mathrm{KPZ}$ equation then reads:

$\partial_{t} h(x, t)=c+\nu \partial_{x}^{2} h(x, t)+\frac{\lambda}{2}\left[\partial_{x} h(x, t)\right]^{2}+\eta(x, t)$,

where $\eta(x, t)$ is an uncorrelated white noise

$$
\left\langle\eta(x, t) \eta\left(x^{\prime}, t^{\prime}\right)\right\rangle=2 D \delta\left(x-x^{\prime}\right) \delta\left(t-t^{\prime}\right) .
$$

The average $\left\langle_{-}\right\rangle$is taken on different realizations of the noise. In Eq.(1) and (2), $c, \nu, \lambda$ and $D$ are coupling parameters ( $c$ is often set to zero because of the invariance of Eq.(11) under rescaling $h \rightarrow h+c t$ ). For $\lambda=0$, Eq.(11) reduces to the Edward-Wilinson (EW) equation [8] which can be solved exactly.

In writing Eq.(1) either a regularization in the correlation given in Eq. (2) (such as for instance a spatially correlated noise ) or the introduction of a minimal length scale $a$ is always tacitly assumed. In the latter case, one is then naturally led to consider a discretization of the continuum equation at a given cutoff length scale $a$. In that case, (a) the noise term $\eta(x, t)$ is discretized

$$
\eta_{i}(t)=\sqrt{\frac{D}{a}} \theta_{i}(t)
$$

where $\theta_{i}(t)$ is a random noise

$$
\left\langle\theta_{i}(t) \theta_{j}\left(t^{\prime}\right)\right\rangle=2 \delta_{i, j} \delta\left(t-t^{\prime}\right) .
$$

with $\delta_{i, j}$ the Kronecker symbol; and (b) Eq. (11) is written for a discrete variable $h_{i}(t)(i=1, \ldots, N=L / a)$ with periodic boundary conditions

$$
\frac{d h_{i}}{d t}=c+\nu_{\mathrm{eff}} F_{i}^{\nu}[h]+\frac{\lambda_{\mathrm{eff}}}{2} F_{i}^{\lambda}[h]+\sqrt{D_{\mathrm{eff}}} \theta_{i}(t) .
$$

Here $\nu_{\text {eff }}=\nu / a^{2}, \lambda_{\text {eff }}=\lambda / a^{2}, D_{\text {eff }}=D / a . \quad F_{i}^{\nu}[h]$ and $F_{i}^{\lambda}[h]$ are proper discretizations of the linear $\partial_{x}^{2} h$ and non-linear $\left(\partial_{x} h\right)^{2}$ terms respectively. We note that the exact meaning of "proper discretization" has been the object of some investigations [16 19].

In all practical applications, a further temporal discretization [15], [20] is also performed on Eq. (5)

$h_{i}(t+\delta t)=h_{i}(t)+\delta t\left(c+\nu_{\mathrm{eff}} F_{i}^{\nu}[h(t)]+\frac{\lambda_{\mathrm{eff}}}{2} F_{i}^{\lambda}[h(t)]\right)+\sqrt{2 D_{\mathrm{ef}}}$

where $r_{i}$ is a Gaussian random generator of unit variance and $\delta t$ the discretization time step.

In $d=1+1$ it is known that the steady state solution $P[h]$ for the probability distribution of the heights in the KPZ equation is identical to the EW stationary distribution due to a fluctuation-dissipation theorem [10]. It was shown [19, 21] that the correct stationary discrete probability namely

$$
P[h]=\mathcal{N}^{-1} \exp \left[-\frac{1}{2} \frac{\nu}{D a} \sum_{i=1}^{N}\left(h_{i}-h_{i+1}\right)^{2}\right],
$$

where $\mathcal{N}^{-1}$ is a normalization factor, can be obtained by taking

$$
F_{i}^{\nu}[h]=h_{i+1}+h_{i-1}-2 h_{i}
$$

and

$F_{i}^{\lambda}[h]=\frac{1}{3}\left[\left(h_{i+1}-h_{i}\right)^{2}+\left(h_{i+1}-h_{i}\right)\left(h_{i}-h_{i-1}\right)+\left(h_{i}-h_{i-1}\right)^{2}\right]$.

The standard choice $F_{i}^{\lambda}[h]=1 / 4\left(h_{i+1}-h_{i-1}\right)^{2}$ on the other hand fails to reproduce Eq. (7) and suffers of other problems as well [18]. A necessary (albeit not sufficient) condition for the identification with the continuum counterpart Eq. (1), is clearly that the correct steady state (i.e. independent of $\lambda$ ) is recovered. For this reason, we shall exploit for the new identification procedure as well as for the LS scheme, equations (8) and (9) hereafter instead of the standard choice which was used in 13 .

\section{THE LEAST SQUARES ERROR MODEL METHOD}

Before introducing our method we first review the LS error method used in Ref. [13]. We consider experimental surfaces coarse-grained at length scale $a$ described by the interface heights $h_{i}^{\text {obs }}(t),(i=1, \ldots, N)$ which are 
sampled $M$ times i.e. at discrete times $t=t_{k}=k \Delta t$ $(k=1, \ldots, M+1)$. Note that the sampling time $\Delta t$ is the time interval between two experimental observations and it is clearly different from the discretization time $\delta t$ of Eq.(6). For surfaces obtained by numerical simulations $\Delta t$ is typically a multiple of $\delta t$. We note that in Ref. [13], the authors used $\Delta t$ equal to $\delta t$ which is a rather particular case.

For the sake of simplicity, we assume here that measurements are free from observational noise. It must be emphasized that, in the presence of measurement noise, our method performs a priori better then the LS scheme since it is based on spatial-averaged values which are less affected by errors on local height measurements.

Our purpose is to determine the coefficients $c, \nu, \lambda, D$ at the given length scale $a$ in Eq.(3).

Let us first neglect the dynamical noise in Eq.([). We then obtain a standard identification problem of the coupling parameters governing a deterministic non-linear equation which can be cast in the compact form:

$$
\frac{d h_{i}}{d t}=\sum_{\alpha=1}^{p} \mu_{\alpha} F_{i}^{\alpha}[h]
$$

where in the present case $p=3$ and $\mu_{1}=c, \mu_{2}=\nu_{\text {eff }}$, $\mu_{3}=\lambda_{\text {eff }}$, (8) and (9) are used for $F_{i}^{2}[h]$ and $F_{i}^{3}[h]$ whereas $F_{i}^{1}[h]=1$.

Optimal parameters are then determined by minimizing a cost function $\mathcal{J}$ such as the sum-square difference

$$
\mathcal{J}=\frac{1}{N M} \sum_{k=1}^{M} \sum_{i=1}^{N}\left[h_{i}^{\mathrm{obs}}\left(t_{k+1}\right)-h_{i}^{\mathrm{pred}}\left(t_{k+1}\right)\right]^{2},
$$

which quantifies the distance between experimental observations $h_{i}^{\text {obs }}\left(t_{k}\right)$ and equivalent reconstructed quantities $h_{i}^{\text {pred }}\left(t_{k}\right)$. The latter quantities are computed from Eq. (10) for given parameters and are thus generally implicit functions of the parameters. However, if the sampling time $\Delta t$ is small enough, then $F_{i}^{\alpha}[h]$ are nearly constant between two measurements and the amplitudes $h_{i}^{\text {pred }}\left(t_{k+1}\right)$ can be related to the parameters $\mu_{\alpha}$ by

$$
h_{i}^{\text {pred }}\left(t_{k+1}\right)=h_{i}^{o b s}\left(t_{k}\right)+\Delta t \sum_{\alpha=1}^{p} \mu_{\alpha} F_{i}^{\alpha}\left[h^{\text {obs }}\left(t_{k}\right)\right]
$$

In this case, the cost function $\mathcal{J}(\{\mu\})$ itself becomes explicit and quadratic in the parameters. Optimal parameters can thus be evaluated through a simple matrix inversion. Indeed the extremal value of $\mathcal{J}$ is inferred by

$$
\left.\frac{\partial \mathcal{J}}{\partial \mu_{\alpha}}\right|_{\mu^{*}}=0
$$

The solution for the optimal parameters $\left\{\mu^{*}\right\}$ is then given by a matrix equation:

$$
\mu_{\alpha}^{*}=\sum_{\beta=1}^{p} A_{\alpha \beta}^{-1} \cdot B_{\beta}
$$

where we have defined

$$
\begin{gathered}
A_{\alpha \beta}=\frac{1}{N M} \sum_{k=1}^{M} \sum_{i=1}^{N} F_{i}^{\alpha} F_{i}^{\beta}, \\
B_{\alpha}=\frac{1}{N M} \sum_{k=1}^{M} \sum_{i=1}^{N}\left[\frac{h_{i}^{o b s}\left(t_{k+1}\right)-h_{i}^{o b s}\left(t_{k}\right)}{\Delta t}\right] F_{i}^{\alpha},
\end{gathered}
$$

and where functions $F_{i}^{\alpha}$ are clearly expressed at $h_{1}^{\mathrm{obs}}\left(t_{k}\right), \ldots, h_{N}^{\mathrm{obs}}\left(t_{k}\right)$.

This classical least squares method is an easy and natural approach and it works fairly well in the absence of any noise. In the presence of noise however, it has its main drawback in the fact that it approximates time derivatives by finite differences. If the dynamics is governed by a deterministic equation and measurements are performed with a negligible observational noise, this simply imposes the choice of a sampling time much smaller than the characteristic or relaxation time of the process.

Lam and Sander 13] assumed that if the sampling time $\Delta t$ is small enough, the above method could be extended to a Langevin equation (i.e. with dynamical noise). The amplitude of the noise can then be inferred from Eq.111) when $J$ is taken at the minimum values of the parameters, that is

$$
\left.D=\frac{1}{2 \Delta t} a J\left(\left\{\mu^{*}\right\}\right)\right)
$$

However it was already observed in dynamical systems that even with pure measurement noise the above method can cause large errors. This is expected to be the case for dynamical noise as well. Two main reasons for this could be advocated. First if $\Delta t$ is too large, the linear approximation (12) which explicitly relates the observed quantities breaks down. Because of the dynamical noise term, this happens a priori for shorter times intervals in a Langevin equation compared with its deterministic counterpart. Second, even in the favorable case in which $\Delta t$ is small, such a method is efficient only if large sizes and small noise amplitudes are used. This is explained in Appendix A where a simple zero-dimensional case is explicitly worked out with the method of Lam and Sander.

\section{STOCHASTIC APPROACH FOR MODEL IDENTIFICATION.}

We now turn to our method which is based on the simple observation that all the information present in the Langevin equation (5) are also contained in the corresponding Fokker-Planck equation (FPE) 15]:

$$
\partial_{t} P[h, t]=\sum_{i=1}^{N} D_{\mathrm{eff}} \frac{\partial^{2}}{\partial h_{i}^{2}} P[h, t]-\sum_{i=1}^{N} \frac{\partial}{\partial h_{i}}\left(F_{i}[h] P[h, t]\right),
$$

where 


$$
F_{i}[h]=c+\nu_{\mathrm{eff}} F_{i}^{\nu}[h]+\frac{1}{2} \lambda_{\mathrm{eff}} F_{i}^{\lambda}[h],
$$

and 22

$$
P[h, t]=\left\langle\prod_{i=1}^{N} \delta\left(h_{i}-h_{i}(t)\right)\right\rangle
$$

where the solution $h_{i}(t)$ is associated to a particular noise configuration $\theta_{i}(t)$.

In equation (18) the second term on the r.h.s. characterizes the deterministic behavior of the system whereas the first term contains stochastics effects. We derive a first general equation involving the parameters $c$ and $\lambda$. Using Eq. (18), the time derivative of the ensemble average of $h_{i}(t)$ can be easily shown to be:

$$
\frac{d\left\langle h_{i}(t)\right\rangle}{d t}=\int \mathcal{D} h F_{i}[h] P[h, t]
$$

where $\mathcal{D} h \equiv \prod_{i=1}^{N} d h_{i}$. If we denote by $g^{(1)}(t)=$ $\frac{1}{N} \sum_{i}\left\langle h_{i}(t)\right\rangle$ the mean height at time $t$ averaged over the noise, its time derivative can be written after some simple algebra as

$$
\frac{d g^{(1)}(t)}{d t}=c+\frac{\lambda_{\mathrm{eff}}}{6}\left[2 g_{0}^{(2)}(t)+g_{1}^{(2)}(t)\right]
$$

where we have defined

$$
g_{l}^{2}(t)=\frac{1}{N} \sum_{i=1}^{N}\left\langle\delta h_{i}(t) \delta h_{i+l}(t)\right\rangle
$$

in the variables $\delta h_{i}=h_{i}-h_{i+1}$. Note that there are only $N-1$ independent $\delta h_{i}$ variables due to periodic boundary conditions and to the fact that $\sum_{i=1}^{N} \delta h_{i}=0$.

The above result prompts a convenient change of variables from $h_{1}, . ., h_{N}$ to $\delta h_{1}, . ., \delta h_{N-1}, \bar{h} \equiv 1 / N \sum_{i=1}^{N} h_{i}$ followed by an integration over $\bar{h}$. Physically this is related to the fact that our system is infinitely degenerate with respect to the average height. Note that the stationary probability Eq. (7) is now Gaussian and well defined in the new variables $\delta h_{1}, \ldots, \delta h_{N-1}$. The corresponding probability $\widetilde{P}[\delta h]$ is the solution of a modified FPE:

$$
\begin{aligned}
\partial_{t} \widetilde{P}[\delta h, t] & =2 D_{\text {eff }} \sum_{i=1}^{N-1} \frac{\partial^{2}}{\partial \delta h_{i}^{2}} \widetilde{P}[\delta h, t] \\
& -\sum_{i=1}^{N-1} \frac{\partial}{\partial \delta h_{i}}\left(G_{i}[\delta h] \widetilde{P}[\delta h, t]\right)-2 D_{\text {eff }} \sum_{i=2}^{N-1} \frac{}{\partial}
\end{aligned}
$$

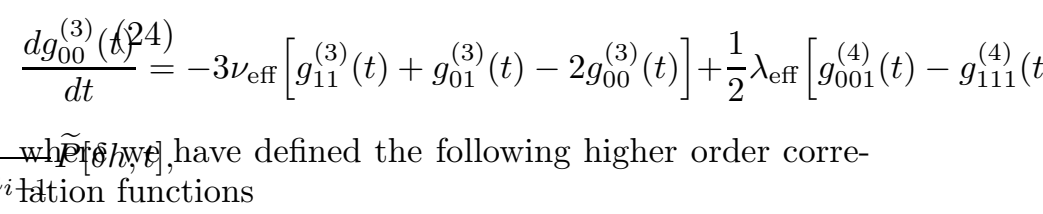

in which $\widehat{\pi}(q, t)$ is the Fourier transform of $\pi\left(\delta h_{j}, t\right)$ averaged over all sites. One can then expand Eq. (32) in powers of $q$ and obtain an infinite hierarchy (closure problem) in the correlation functions. The first two nontrivial orders $\left(O\left(q^{2}\right)\right.$ and $\left.O\left(q^{3}\right)\right)$ are

$$
\frac{d g_{0}^{(2)}(t)}{d t}=4 \nu_{\mathrm{eff}}\left[g_{1}^{(2)}(t)-g_{0}^{(2)}(t)\right]+4 D_{\mathrm{eff}}
$$

and

$$
g_{l m}^{(3)}(t)=\frac{1}{N} \sum_{i=1}^{N}\left\langle\delta h_{i}(t) \delta h_{i+l}(t) \delta h_{i+m}(t)\right\rangle,
$$

$$
G_{i}=F_{i}-F_{i+1}=\nu_{\mathrm{eff}} G_{i}^{\nu}+\frac{1}{2} \lambda_{\mathrm{eff}} G_{i}^{\lambda},
$$

with

$$
G_{i}^{\nu}=\delta h_{i+1}+\delta h_{i-1}-2 \delta h_{i}
$$

$g_{l m n}^{(4)}(t)=\frac{1}{N} \sum_{i=1}^{N}\left\langle\delta h_{i}(t) \delta h_{i+l}(t) \delta h_{i+m}(t) \delta h_{i+n}(t)\right\rangle$ 
It is worth mentioning that $\lambda$ does not explicitly appear in equation (33). As one can explicitly check, this is a feature associated to the particular discretization Eq. (9) and it would have not been the case had we used the standard discretization for $F_{i}^{\lambda}[h]$. This is clearly in turn related to the fact that the steady state probability distribution Eq. (7) is independent of $\lambda$. We also note that in the $1+1$ case we are considering, the explicit steady state solution of Eq.(32) is known, and depends only on a single parameter $\frac{D}{\nu}$. As a consequence, the steady version of Eq. (32) cannot be used here to identify $\nu$ and $D$. In the $2+1$ case where such a peculiar feature is not present, the stationary solution depends on $\lambda$ as well and parameters identification can exploit the steady analogue equation.

\section{PARAMETERS IDENTIFICATION}

Our aim is to implement an identification procedure which could be exploited on real experiments. For this reason, we assume that the experimental surface is constituted by a finite number of sites $N$ with lattice spacing $a$ (corresponding to a size $L=N a$ ), and it is measured during a finite time $T_{\text {obs }}$ every sampling time $\Delta t$. Again $\Delta t$ is a priori different from the discretization time $\delta t$ when the data is produced numerically (note that in real experiments $\delta t$ is not even defined!). We shall test the robustness and efficiency of the scheme with respect to the size $L$ and sampling time $\Delta t$.

Identification methods are often based on minimizing a cost function defined through dynamical constraints. This is clearly the case of the least square method as explained in section III. Here we derive dynamical constraints using Eqns. (22) and (33) which contain informations of the original Langevin equation including mean values and fluctuations around mean values. The present identification is thus based on deterministic equations. This constitutes a crucial difference with respect to the previous reconstruction method [13] directly based on stochastic equations. Another important feature is that the observed quantities we use in our reconstruction scheme are dealing with averaged site values. Hence the fluctuations of all these terms, which derive from stochastic quantities, are reduced typically by a factor $1 / \sqrt{N}$, and self-averaging is expected to be more effective.

Let us derive the constraints we use. First, the total observation time $T_{o b s}$ is divided into $q$ equal slices $\left[T_{1}, T_{2}\right], \ldots,\left[T_{q}, T_{q+1}\right]$ with $\Delta T=T_{j+1}-T_{j}$. Let us integrate (22) and (33) on each slice $\left[T_{j}, T_{j+1}\right]$ :

$$
\frac{\Delta g^{(1)}}{T_{j+1}-T_{j}}=c+\frac{\lambda_{\mathrm{eff}}}{6} \frac{1}{T_{j+1}-T_{j}} \int_{T_{j}}^{T_{j+1}} d t\left[2 g_{0}^{(2)}(t)+g_{1}^{(2)}(t)\right]
$$
t) and3parameters $\nu=D=1$ and $\lambda=3$. These are the $\frac{\Delta g_{0}^{(2)}}{T_{j+1}-T_{j}}=4 \nu_{\mathrm{eff}} \frac{1}{T_{j+1}-T_{j}} \int_{T_{j}}^{T_{j+1}} d t\left[g_{1}^{(2)}(t)-g_{0}^{(2)}(t)\right]+4 \begin{aligned} & \text { lems and the non-linear term } \lambda \text { is big enough to be well } \mathrm{KPZ} \text { phase. We find interesting to repeat each } \\ & \text { calculation few times (typically } 5) \text { to give an estimate of }\end{aligned}$ same values used in Ref. [14. The time step is expected
to be sufficiently small for not causing instability probto be sufficiently small for not causing instability prob-

If functions and integrals in Eqns. (37) and (38) are computed using experimental data, these discrete equations provide $2 q$ relations between the parameters to identify. From these constraints, two cost functions are built in a way already described in Sec. III with $p=2$. The corresponding $2 \times 2$ equations then yield $c$ and $\lambda$ from one cost function and $\nu$ and $D$ from the other.

We now explain how functions and integrals in Eqns. (37) and (38) are obtained experimentally. Starting with a same initial surface e.g. a flat surface, we will grow the surface $\mathcal{R}$ times. Because of the stochastic nature of the phenomenon, this produces $\mathcal{R}$ different observations or realizations of the same process . Such a procedure, which can be performed very easily in real experiments, allows the computation, at sampling times $t=t_{k}=k \Delta t$ $(k=1, \ldots, M+1)$, of $\left[g^{(1)}\right]_{\exp },\left[g_{0}^{(2)}\right]_{\exp }$ and $\left[g_{1}^{(2)}\right]_{\exp }$. Indeed, these quantities are the averages over $\mathcal{R}$ different realizations of the spatial average height and correlations of the first neighbors. The number $\mathcal{R}$ of realizations need not be large: if the total number $N$ of sites is sufficiently large, the experimental values are rather close to the corresponding theoretical predictions $g^{(1)}(t)$, $g_{0}^{(2)}(t)$ and $g_{1}^{(2)}(t)$. From these functions sampled every $t=t_{k}=k \Delta t$, integrals in Eqns. (37) and (38) can be efficiently evaluated for small sampling time $\Delta t$. In this case, the smooth functions $\left[g^{(1)}\right]_{\exp },\left[g_{0}^{(2)}\right]_{\exp }\left[g_{1}^{(2)}\right]_{\exp }$ can be approximated on the whole time interval $\left[T_{1}, T_{q+1}\right]$ by a standard curve fitting algorithm which gives as a by product the time integrals. This method does impose a constraint on the sampling time $\Delta t$. However, this constraint is substantially weaker with respect to that imposed by the LS method as we will show below. This is a considerable advantage of our new procedure.

Two remarks are here in order. Firstly one expects the result to be independent on the number of slices $q$ provided that $q$ satisfies the following two constraints. On the one hand $q$ should be greater than 2 (since two parameters are identified per cost functions) and on the other hand it should be less than $M=\frac{T_{o b s}}{\Delta t}$ so that $\Delta T$ cannot be less than the sampling time $\Delta t$. Secondly the identification of $\nu$ and $D$ could be achieved by using Eq. (32) rather than Eq. (33). We shall see that in our approach the two equations yield virtually identical results.

\section{RESULTS}

In order to test the potentiality of the different identification methods, we produce experimental data by simulating Eq.(5) with a standard Euler time integration algorithm with time step $\delta t=0.01$, lattice spacing $a=1$, nside the KPZ phase. We find interesting to repeat each 
the error bars to be associated to each parameter value (this was missing in previous works).

\section{A. LS Method}

Let us compute the parameters using the original LS method with the spatial and temporal discretization Eqns. (5) and (6). We exploit the same trick used in Ref. 14 in which a KPZ surface of size $2 L$ is obtained by a magnification of a fully relaxed surface of size $L$ where the height are rescaled by a factor $2^{\alpha},(\alpha=0.5)$ and linearly interpolated. The obtained surface is then relaxed to stationarity before a successive magnification is attempted. However, unlike Ref. [14] where a single surface of size $L=32768$ was computed, we consider $L=512,1024,2048,4096$ and linearly extrapolate the results to the limit $L \rightarrow \infty$. The calculation is repeated for increasing values of $s=\Delta t / \delta t$ in order to display the crucial weakness of the method as explained before. Fig. 11 depicts the results for the parameter $\nu$ at finite $L$. Similar trends are present for $\lambda$ and $D$. The extrapolated values at $L \rightarrow \infty$ are reported in Table If. The gradual decrease in the precision of the reconstructed parameters is apparent and it shows the loss of accuracy of the LS method as $\Delta t$ increases as previously advertised.

We also considered the LS when the reconstructed quantities are computed at time intervals $\Delta T$ which are multiple of the sampling time $\Delta t$. In fact this test was also carried out by the authors of Ref. [14] (in their notations $\tau=\Delta T$ and $\Delta t=\delta t$ ) and it will constitute a further source of comparison with our alternative stochastic method (see below). Even in this case there is a decrease in the performance of the procedure as the ratio $r=\Delta T / \Delta t$ increases, consistently with the results of Ref. [14]. The corresponding extrapolated values are reported in Table II.

\section{B. Stochastic Approach}

For a more convenient comparison with the LS method, we use the same sizes and statistics (five different configurations for each size). Our calculations are carried out in the transient rather than in the steady state and are therefore much less time consuming. Again the results are obtained for $L=512,1024,2048,4096$ and linearly extrapolated to $L \rightarrow \infty$. For a comparison with the previous calculation, the outcomes of the parameter $\nu$ at different size $L$ are plotted in Fig.2 for increasing values of the ratio $s=\Delta t / \delta t$, and the corresponding extrapolated values are reported in Table III. One can see that the parameter values are rather insensitive to the changing the ratio $s=\Delta t / \delta t$ as expected. Next we check the performance of our method with respect to the increasing of the ratio $r=\Delta T / \Delta t$. This is reported in Table
IV. As expected, our method outperforms the LS one in all situations.

Since the LS method could in principle be carried out in transient rather than in steady state conditions, one might wonder how it would perform in this case.

To this aim we recompute the parameters using the LS method under these conditions and find that the predicted values are far off with respect to the exact ones. For instance for $L=4096$ a typical run yields $\nu \sim 0.36$, $\lambda \sim 0.68$ and $D \sim 0.005$ to be compared with a typical result obtained with our method $\nu \sim 0.99, \lambda \sim 2.98$ and $D \sim 1.01$.

As a final cross-check of our method, we recompute the parameters in the same situation as before but using Eq. (32) rather than Eq. (33) to extract $\nu$ and $D$ and find nearly identical values.

\section{Coarse-graining and KPZ real discretization.}

The application of this machinery to experimental surfaces assumes that the system is described by a KPZ-like dynamics. In this case, besides being able to address the issue of whether or not they belong to the KPZ universality class, one would be able to provide a numerical estimates of the coupling parameters which are usually overlooked in studies focussing only on the universality class.

Following Lam and Sander [13], we produce an interface based on the KPZ discretized model Eq. (5) which is then smoothed by introducing the (discrete) Fourier transform of the heights

$$
\widehat{h}_{q_{n}}(t)=a \sum_{i=1}^{N} e^{-\mathrm{iq}_{\mathrm{n}} \mathrm{x}_{\mathrm{i}}} h_{i}(t)
$$

A coarse-graining surface at level $a_{s}=b a$ can then be achieved by simplying setting to zero all wavelength components $\widehat{h}_{q_{n}}(t)$ with $q \geq N_{s}=L / a_{s}$ and transforming back to real space. The obtained smoothed surface is then assumed to be governed by a KPZ equation (renormalizability property). This new KPZ dynamics can then reconstructed along lines similar to those described above before a further time step is carried out on the original surface.

With $b=2, \Delta T / \Delta t=1$ and sizes up to $L=8192$ averaged over 5 configurations as before, we find $\nu=$ $1.09 \pm 0.04, \lambda=3.27 \pm 0.05$, and $D=0.88 \pm 0.03$. Higher values of $b$ result in poorer and poorer agreement with the expected values even with higher lattice sizes. The same feature is also present in the original LS procedure as we explicitly checked. In fact this is a general deficiency of the real space discretization as explained in Appendix B: the finite size difference has lost some renormalizability property of the original KPZ continuum equation. 


\section{CONCLUSIONS}

In this paper, we discuss a method for extracting the coupling parameters from a non-linear Langevin equation starting from experimental surfaces representing successive snapshots of the system. We apply this scheme to the KPZ equation in $1+1$ dimensions (although it could be extended to any dimensions) and compare it with the previous approach of Ref. [13], finding the following differences. First of all it does not require large sizes and it is well suited for a transient state. This is expected to be a considerable advantage, notably in numerical work, since the typical time required to reach a steady state increases as $L^{z}$ where $z$ is the dynamical exponent $(3 / 2$ in the $1+1 \mathrm{KPZ}$ case). We have explicitly shown how the LS method which works rather well in the aforementioned conditions, fails to provide sensible answers otherwise. Most importantly however is the fact that our approach is stable under the changing of the sampling time, unlike the LS method which is not. We stress the importance of this feature since in typical experimental situations, the sampling time is an externally tuned parameters which has nothing to do with the evolution time of the system. We have discussed the reasons why this is so and provide an intuitive heuristic argument showing why the LS scheme is not expected to work under these more realistic conditions. Finally we implemented a coarse-graining procedure in order to be able to apply our method to experimentally generated profiles. We showed that the agreement with the expected values is much poorer in the present case and we further argued that any real space based approach is doomed to run into this problem, the reason being that they have not a correct renormalization behavior under coarse-graining as explained in Appendix B. We have recently devised an alternative approach based on a Fourier-based scheme which avoids discretization problems. The results of this will be the subject of a forthcoming publication.

\section{ACKNOWLEDGMENTS}

This work was supported by a joint CNR-CNRS exchange program number 5274. One of us (AG) acknowledges financial support by MURST and INFM.

\section{APPENDIX A: A SIMPLE SOLVABLE EXAMPLE}

This appendix shows, on a simple and solvable example which is a zero-dimensional analogue of Eq.(11), that the method of least squares can be hampered by the presence of dynamical noise. Let us assume that the scalar variable $X(t)$ is governed by the following Langevin equation:

$$
\frac{d X}{d t}=B(X)+\mu G(X)+\eta(t)
$$

where $B$ and $G$ are prescribed functions, $\eta(t)$ is an uncorrelated white noise

$$
\left\langle\eta(t) \eta\left(t^{\prime}\right)\right\rangle=2 D \delta\left(t-t^{\prime}\right)
$$

For simplicity, we assume that: (a) measurement noise is negligible, (b) the observed time serie $X^{\text {obs }}\left(t_{k}\right)$ with $t_{k}=k \Delta t(k=1, \ldots, M+1)$ has been produced by the dynamical system (A1) with the value $\mu=0$. We ask whether the least square method is capable of identifying the correct coupling parameter $\mu=0$.

From the one hand, the least square method first assumes that the data is produced by the deterministic counterpart of Eq. (A1) with an unkown parameter $\mu$. In discrete times, this yields a "predicted" value given by

$$
X^{\text {pred }}\left(t_{k+1}\right)=X^{\text {obs }}\left(t_{k}\right)+\Delta t\left[B\left(X^{\mathrm{obs}}\left(t_{k}\right)\right)+\mu G\left(X^{\text {obs }}\left(t_{k}\right)\right)\right] \text {. }
$$

On the other hand, the "observed" value is given, if the sampling time is small enough, by the discrete time counterpart of Eq.(A1) with $\mu=0$ :

$$
X^{\mathrm{obs}}\left(t_{k+1}\right)=X^{\mathrm{obs}}\left(t_{k}\right)+\Delta t B\left(X^{\mathrm{obs}}\left(t_{k}\right)\right)+r\left(t_{k}\right) \sqrt{2 D \Delta t},
$$

where $r\left(t_{k}\right)$ is a gaussian random generator of unit variance.

Using both experimental observations $X^{\text {obs }}\left(t_{k}\right)$ and reconstructed quantities $X^{\text {pred }}\left(t_{k}\right)$, a cost function can be constructed:

$$
\mathcal{J}=\frac{1}{M} \sum_{k=1}^{M}\left[X^{\mathrm{obs}}\left(t_{k+1}\right)-X^{\mathrm{pred}}\left(t_{k+1}\right)\right]^{2} .
$$

Using Eqns. (A3) and (A4), the cost function is readly rewritten as

$$
\mathcal{J}=\frac{1}{M} \sum_{k=1}^{M}\left[\Delta t \mu G\left(X^{\mathrm{obs}}\left(t_{k}\right)\right)-r\left(t_{k}\right) \sqrt{2 D \Delta t}\right]^{2} .
$$

The minimum value of $\mathcal{J}$ then satisfies the extremality condition

$$
\left.\frac{\partial \mathcal{J}}{\partial \mu}\right|_{\mu^{*}}=0
$$

which provides the value

$$
\mu^{*}=\sqrt{\frac{2 D}{\Delta t}} \frac{\frac{1}{M} \sum_{k=1}^{M} r\left(t_{k}\right) G\left(X^{\mathrm{obs}}\left(t_{k}\right)\right)}{\frac{1}{M} \sum_{k=1}^{M} G^{2}\left(X^{\mathrm{obs}}\left(t_{k}\right)\right)} .
$$

Let us estimate the two sums appearing in Eq.(A\&). Because of self-averaging, the denominator can be clearly rewritten as

$$
\frac{1}{M} \sum_{k=1}^{M} G^{2}\left(X^{\mathrm{obs}}\left(t_{k}\right)\right) \approx\left\langle G^{2}\right\rangle
$$

the average being over the noise $\eta$. The second sum in can be separated into two contributions 
$\frac{1}{M} \sum_{k=1}^{M}\left[r\left(t_{k}\right) G\left(X^{\text {obs }}\left(t_{k}\right)\right]=\frac{1}{M} \sum_{k=1}^{M} r\left(t_{k}\right)\left[G\left(X^{o b s}\left(t_{k}\right)-\langle G\rangle\right]\right.\right.$ it

where using the Central Limit Theorem (CLT) [4 we have that

$$
\frac{\langle G\rangle}{M} \sum_{k=1}^{M} r\left(t_{k}\right) \approx \frac{\langle G\rangle}{\sqrt{M}} r_{1}
$$

where $r_{1}$ is a random variable of unit variance. In Eq.(A10), each term of the first sum of the r.h.s. is a random variable which is a product of two independent random variables of zero mean and variance respectively equal to 1 and $\sigma=\left\langle G^{2}\right\rangle-\langle G\rangle^{2}$. A similar argument based on the CLT applies to the evaluation of the first term of the r.h.s. since it is again a sum of $M$ random variables with zero average which implies that:

$$
\frac{1}{M} \sum_{k=1}^{M} r\left(t_{k}\right)\left[G\left(X^{\mathrm{obs}}\left(t_{k}\right)-\langle G\rangle\right] \approx \frac{\sqrt{\sigma}}{\sqrt{M}} r_{2}\right.
$$

where $r_{2}$ is a random variable of unit variance. Instead of the true value one then finds

$$
\mu^{*} \approx \frac{\sqrt{2 D}}{\sqrt{M \Delta t}} \frac{\operatorname{Max}(\sqrt{\sigma},\langle G\rangle)}{\left\langle G^{2}\right\rangle} .
$$

With non-vanishing amplitudes $D$, the noise hence causes errors for small $\Delta t$ unless large statistics are considered.

\section{APPENDIX B: NON-RENORMALIZABILITY OF REAL SPACE DISCRETIZATIONS}

Let us consider a one-dimensional surface defined on a lattice of length $L=N a$ ( $a$ being the lattice spacing and $N$ being the total number of sites) which is identified by $h_{1}, \ldots, h_{N}$ at positions $x_{1}=a, \ldots, x_{N}=N a$ and having periodic boundary conditions. If we assume a KPZ dynamics, then the equation of motion is given by Eq.(5) and its stationary state by Eq. (7). Let us introduce the (discrete) Fourier transform $\widehat{h}_{q}$ so that

$$
h_{i}(t)=\frac{1}{L} \sum_{n=-N / 2}^{N / 2} e^{\mathrm{i} \mathrm{q}_{\mathrm{n}} \mathrm{x}_{\mathrm{i}}} \widehat{h}_{q_{n}}(t)
$$

and conversely

$$
\widehat{h}_{q_{n}}(t)=a \sum_{i=1}^{N} e^{-\mathrm{i} \mathrm{q}_{\mathrm{n}} \mathrm{x}_{\mathrm{i}}} \cdot h_{i}(t)
$$

By using the relation

$$
\sum_{i=1}^{N} e^{\mathrm{i}\left(\mathrm{q}_{\mathrm{n}}-\mathrm{q}_{\mathrm{m}}\right) \mathrm{x}_{\mathrm{i}}}=N \delta_{n,-m}
$$

$$
\widehat{P_{a}}[\widehat{h}]=\mathcal{N}^{-1} \exp \left[-\frac{1}{2} \frac{\nu}{D L} \sum_{n=-N / 2}^{N / 2} q_{n}^{2}\left|\widehat{h}_{q_{n}}\right|^{2}\right] .
$$

In Eq.(B4) the continuum limit $a \rightarrow 0$ is simply achieved by letting $N \rightarrow \infty$.

We now recall that the proper variables to be used in this context are the $\delta h_{i}=h_{i}-h_{i+1}$ whose Fourier transform $\delta \widehat{h}_{q_{n}}$ are related to the Fourier transform $\widehat{h}_{q_{n}}$ of the heights by

$$
\delta \widehat{h}_{q_{n}}=\widehat{h}_{q_{n}}\left(1-e^{\mathrm{i} \mathrm{q}_{\mathrm{n}} \mathrm{a}}\right) .
$$

We can exploit the periodic boundary conditions to extend the $N-1$ sites to $N$ (bearing however in mind that only $N-1$ are independent) and use the the fact that

$$
\frac{1}{a} \sum_{i=1}^{N} \delta h_{i}^{2}=\frac{1}{L a^{2}} \sum_{n=-N / 2}^{N / 2}\left|\delta \widehat{h}_{q_{n}}\right|^{2}
$$

to rewrite the probability (B4) in the alternative form

$$
\widehat{P_{a}}[\widehat{h}]=\mathcal{N}^{-1} \exp \left[-\frac{1}{2} \frac{\nu}{D L a^{2}} \sum_{n=-N / 2}^{N / 2}\left|\widehat{h}_{q_{n}}\left(1-e^{\mathrm{i} \mathrm{q}_{\mathrm{n}} \mathrm{a}}\right)\right|^{2}\right] .
$$

In the continuum limit $a \rightarrow 0$ one can explicitly check that Eq.(B7) is equivalent to Eq. (B4) by expanding the term $e^{\mathrm{iqn} a}$ in powers of $a$ and keeping the lowest nonvanishing order $O(a)$. Nevertheless Eq.(B7) leads to problems when one tries to coarse grain the surface. Indeed suppose we now perform a smoothing of the lattice of a factor $b$ to obtain a new lattice constant $a_{s}=b a$ and a decreased number of sites $N_{s}=N / b$. This amounts to set to zero all modes from $N_{s}$ to $N$ and thus the correct corresponding stationary distribution is according to (B7)

$\widehat{P_{a_{s}}}[\widehat{h}]=\mathcal{N}_{\mathrm{s}}^{-1} \exp \left[-\frac{1}{2} \frac{\nu}{D L a^{2}} \sum_{n=-N_{s} / 2}^{N_{s} / 2}\left|\widehat{h}_{q_{n}}\left(1-e^{\mathrm{i} \mathrm{q}_{\mathrm{n}} \mathrm{a}}\right)\right|^{2}\right]$.

On the other hand, had we started from the "smoothed" lattice and construct the differences $\delta h_{i}^{\mathrm{s}}=$ $h_{i}^{\mathrm{s}}-h_{i+b}^{\mathrm{s}}$ of the coarse-grained heights, we would have found

$\widehat{P_{a_{s}}}[\widehat{h}]=\mathcal{N}_{\mathrm{s}}^{-1} \exp \left[-\frac{1}{2} \frac{\nu}{D L a_{s}^{2}} \sum_{n=-N_{s} / 2}^{N_{s} / 2}\left|\widehat{h}_{q_{n}}\left(1-e^{\mathrm{iq} \mathrm{b} \mathrm{ba}}\right)\right|^{2}\right]$,

which differs from the previous one.

It is clear that this is a general problem of all discretizations in real space. In the limit $a \rightarrow 0$ in fact, one recovers from (B9) the correct expression (B4). 


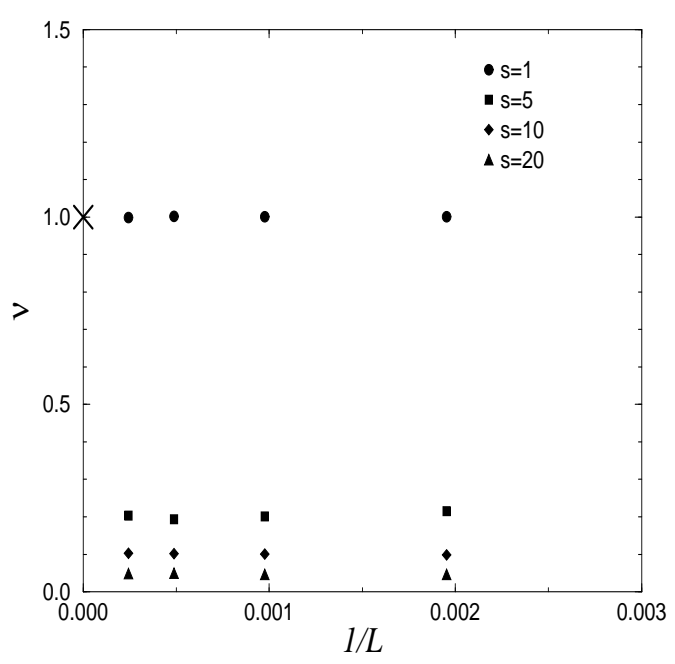

FIG. 1. The coupling parameter $\nu$ for increasing lattice sizes $L=512,1024,2048,4096$, in the original steady-state LS method. All quantities are in dimensionless form. Error bars are of order of the symbol sizes and are consequently not displayed. Different curves refer to increasing values of the ratio $s=\Delta t / \delta t$. The cross $(\mathrm{X})$ indicates the exact value of the parameter $\nu=1$.

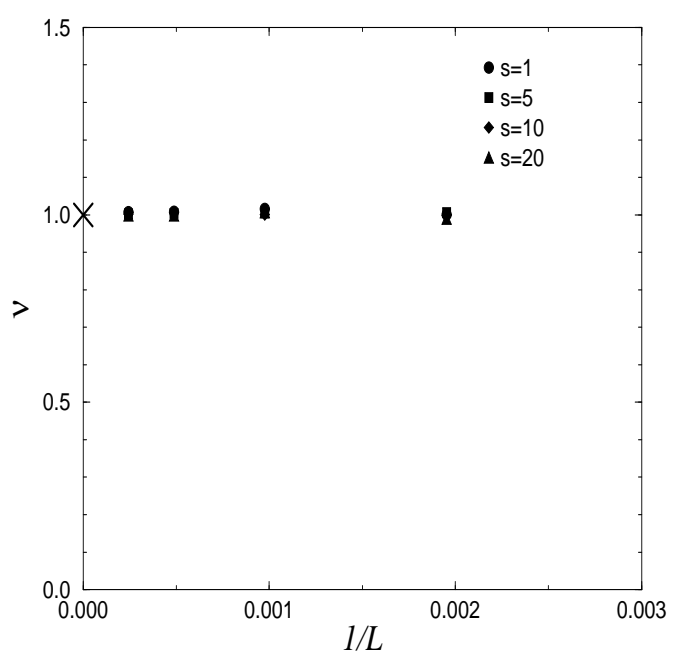

FIG. 2. The coupling parameter $\nu$ for increasing lattice sizes $L=512,1024,2048,4096$, as obtained from our reconstruction method. Error bars are of order of the symbol sizes and are consequently not displayed. Different curves refer to increasing values of the ratio $s=\Delta t / \delta t$. The cross (X) indicates the exact value of the parameter $\nu=1$.

TABLE I. Extrapolated values of coupling parameters $\nu(\infty), \lambda(\infty)$ and $D(\infty)$ as a function of $s=\Delta t / \delta t$ (see text) as computed from the original LS method (at steady state).

\begin{tabular}{lccc}
$\Delta t / \delta t$ & $\nu(\infty)$ & $\lambda(\infty)$ & $D(\infty)$ \\
\hline 1 & $0.999 \pm 0.003$ & $2.980 \pm 0.005$ & $1.000 \pm 0.001$ \\
5 & $0.194 \pm 0.004$ & $0.595 \pm 0.015$ & $0.200 \pm 0.001$ \\
10 & $0.103 \pm 0.004$ & $0.319 \pm 0.008$ & $0.100 \pm 0.001$ \\
20 & $0.049 \pm 0.002$ & $0.139 \pm 0.004$ & $0.050 \pm 0.001$ \\
\hline \hline
\end{tabular}

TABLE II. Extrapolated values of coupling parameters $\nu(\infty), \lambda(\infty)$ and $D(\infty)$ as a function of $r=\Delta T / \Delta t$ (see text) as computed from the original LS method(at steady state).

\begin{tabular}{lccc}
\hline \hline$\Delta T / \Delta t$ & $\nu(\infty)$ & $\lambda(\infty)$ & $D(\infty)$ \\
\hline 1 & $0.999 \pm 0.003$ & $2.980 \pm 0.005$ & $1.000 \pm 0.001$ \\
5 & $0.996 \pm 0.002$ & $2.817 \pm 0.004$ & $0.928 \pm 0.001$ \\
10 & $0.917 \pm 0.002$ & $2.627 \pm 0.006$ & $0.853 \pm 0.001$ \\
20 & $0.855 \pm 0.002$ & $2.308 \pm 0.006$ & $0.757 \pm 0.001$ \\
50 & $0.650 \pm 0.001$ & $1.558 \pm 0.006$ & $0.653 \pm 0.002$ \\
\hline \hline
\end{tabular}

TABLE III. Extrapolated values of coupling parameters $\nu(\infty), \lambda(\infty)$ and $D(\infty)$ as a function of $s=\Delta t / \delta t$ as computed from our stochastic approach in the transient state.

\begin{tabular}{lccc}
\hline \hline$\Delta t / \delta t$ & $\nu(\infty)$ & $\lambda(\infty)$ & $D(\infty)$ \\
\hline 1 & $1.009 \pm 0.002$ & $3.047 \pm 0.016$ & $1.026 \pm 0.001$ \\
5 & $1.008 \pm 0.007$ & $3.015 \pm 0.006$ & $1.003 \pm 0.007$ \\
10 & $1.035 \pm 0.011$ & $2.993 \pm 0.010$ & $1.018 \pm 0.011$ \\
20 & $0.997 \pm 0.020$ & $3.001 \pm 0.005$ & $0.978 \pm 0.010$ \\
\hline \hline
\end{tabular}

TABLE IV. Extrapolated values of coupling parameters $\nu(\infty), \lambda(\infty)$ and $D(\infty)$ as a function of $r=\Delta T / \Delta t$ as computed from our stochastic approach in the transient state.

\begin{tabular}{lccc}
\hline \hline$\Delta T / \Delta t$ & $\nu(\infty)$ & $\lambda(\infty)$ & $D(\infty)$ \\
\hline 1 & $1.009 \pm 0.002$ & $3.047 \pm 0.016$ & $1.026 \pm 0.001$ \\
5 & $1.003 \pm 0.003$ & $3.005 \pm 0.010$ & $1.057 \pm 0.003$ \\
10 & $1.003 \pm 0.001$ & $3.030 \pm 0.007$ & $1.023 \pm 0.001$ \\
20 & $0.998 \pm 0.002$ & $3.014 \pm 0.009$ & $1.016 \pm 0.001$ \\
50 & $0.991 \pm 0.003$ & $3.017 \pm 0.006$ & $1.010 \pm 0.003$ \\
\hline \hline
\end{tabular}


[1] A.S. Weigend and N.A. Gershenfeld, Time Series Prediction, Addison Wesley, (1994).

[2] H.D. Abarbanel, R. Brown, J. J. Sidorowitch and LS Tsimring, Rev. Mod. Phys. 65, 1331 (1993).

[3] E. J. Kostelich and T. Schreiber, Phys. Rev. E 48, 1752 (1993).

[4] See e.g. C. W. Gardiner Handbook of Stochastic Methods, 2nd edition (Springer Verlag 1990); N. G. van Kampen Stochastic processes in Physics and Chemistry (NorthHolland 1992).

[5] L. Battiston and M. Rossi, Int. Journal of Chaos and Applications, (to appear)

[6] M. Kardar, G. Parisi and Y. C. Zhang, Phys. Rev. Lett. 56, 889 (1986).

[7] For recent reviews see e.g. J. Krug, Adv. Phys. 46, 139 (1997); A.L. Barabasi, H. E. Stanley, Fractal Concepts in Surface Growth (Cambridge University Press, Cambridge 1995); T. Halpin-Haley and Y. C. Zhang, Phys. Rep. 254, 215 (1995); M. Marsili, A. Maritan, F. Toigo and J. R. Banavar, Rev. Mod. Phys. 68, 963 (1996); P. Meakin, Phys. Rep. 235, 131 (1993);

[8] S. F. Edward and D. R. Wilkinson, Proc. R. Soc. London A 381, 17 (1982).

[9] D. A. Huse and C. Henley, Phys. Rev. Lett. 54, 2708 (1985).

[10] D. Forster, D. R. Nelson and M. J. Stephen, Phys. Rev. A 16, 732 (1977).

[11] V. Yakhot, Phys. Rev. A 24, 642 (1981).

[12] B. Bogosian, C. C. Chow and T. Hwa, condmat/9911069.

[13] C. Lam and L.M. Sander, Phys.Rev. Lett. 71 , 561, (1993).

[14] C. Lam and F.G. Shin, Phys.Rev. E 58 ,5592 (1998).

[15] H. Risken, The Fokker-Planck Equation, Springer-Verlag, Berlin, (1989).

[16] M. Beccaria and G. Curci, Phys. Rev. E 50, 4560 (1994)

[17] T. J. Newman and M. R. Swift, Phys. Rev. Lett. 79, 2261 (1997)

[18] T. J. Newman and A. J. Bray, J. Phys. A 29, 7917 (1996).

[19] C. Lam and F.G. Shin, Phys.Rev. E 57 ,6506 (1998).

[20] R.Mannella, Computer experiments in non-linear stochastic physics, in: Noise in nonlinear dynamical systems, vol. 3, ed. by F. Moss, P.V.E. McClintock, Cambridge University Press, Cambridge, (1989).

[21] A correct discretization was also previously obtained by A. Maritan (private communication).

[22] See e.g. N. Goldenfeld Lectures on Phase Transitions and the Renormalization Group (Addison-Wesley 1993) pg. 226. 